\title{
SYMPTOMS AND HEALTH COMPLAINTS AND THEIR ASSOCIATION WITH PERCEIVED STRESS AT UNIVERSITY: SURVEY OF STUDENTS AT ELEVEN FACULTIES IN EGYPT
}

\author{
Walid El Ansari', Reza Oskrochi' ${ }^{2}$, Shokria Labeeb ${ }^{3}$, Christiane Stock ${ }^{4}$ \\ ${ }^{1}$ Faculty of Applied Sciences, University of Gloucestershire, Gloucester, United Kingdom \\ ${ }^{2}$ Faculty of Technology, Design and Environment, Oxford Brookes University, Oxford, United Kingdom \\ ${ }^{3}$ Faculty of Nursing, Assiut University, Assiut, Egypt \\ ${ }^{4}$ Unit for Health Promotion Research, University of Southern Denmark, Esbjerg, Denmark
}

\section{SUMMARY}

Aims: This study examined the relationships between perceived stress and a range of self reported symptoms and health complaints in a representative sample of students at one university in Egypt.

Methods: The data (3,271 students) was collected during the academic year 2009-2010 at eleven faculties at the university of Assiut city, Egypt. A self-administered questionnaire measured health complaints (22 symptoms) and Cohen's Perceived Stress Scale. Socio-demographic and lifestyle data were also collected. Factor analysis generated four groups of health complaints: psychological, circulatory/breathing, gastrointestinal, and pains/aches, and the internal consistency of each group of symptoms was computed using reliability analyses (Cronbach's alpha). Perceived stress was categorized into four levels based on quartiles. Multiple binomial or multinomial logistic regression analyses analysed the relationship between each of the four groups of symptoms and other students' general characteristics adjusted for the effect of all other groups of symptoms.

Results: The symptoms most often reported as having occurred sometimes/very often in the last 12 months were fatigue (85.3\%), difficulties to concentrate $(78.1 \%)$, headache $(77.9 \%)$, and mood swings $(74.5 \%)$, while nervousness/anxiety $(63.2 \%)$ and sleep disorder $(63.7 \%)$ affected many students. Multinominal logistic regression revealed a clear association and a linear trend between increasing level of stress and a higher frequency of psychological symptoms that remained significant after adjustment for other groups of symptoms. There were no associations between perceived stress and circulatory/breathing symptoms, gastrointestinal symptoms, or for pains/aches. Poor health was consistently associated with higher frequency of symptoms across all symptom groups except for gastrointestinal symptoms. Higher health awareness was associated with lower frequency of psychological and circulatory/breathing symptoms but not for the other two symptom groups. Better quality of life was associated with lower frequency of psychological and partly for circulatory/breathing symptoms, but not for the other two symptom groups. There were differences in levels of psychological symptoms and of perceived stress between faculties, where students of Physical Education showed the lowest risk profile regarding both psychological symptoms and perceived stress, while students of Veterinary Medicine showed the highest risk profile. Across all four symptom groups, females had higher ratings of complaints than males.

Conclusions: The high prevalence of health complaints especially of psychological and pains/aches type of symptoms raise concern and calls for preventive actions at universities. Comprehensive programmes would be recommended that take the co-occurence of perceived stress and health complaints into account.

Key words: self reported symptoms, health complaints, stress, quality of life, university students, college health

Address for correspondence: W. El Ansari, Faculty of Applied Sciences, University of Gloucestershire, Oxstalls Campus, Oxstalls Lane, Gloucester GL2 9HW, United Kingdom. E-mail: walidansari@glos.ac.uk

\section{INTRODUCTION}

Health complaints of university students are becoming an increasing concern to educators, campus managers and health authorities alike. Published studies consistently highlighted the importance of a wide range of health complaints pertaining to college students, e.g., in Ireland, Korea, Singapore, Nigeria, and Oman (1-5).

University students feel a wide assortment of symptoms, referred to as self-reported symptoms, health complaints, psychosomatic symptoms, or health strains or burdens. Such complaints include: back pains and shoulder pains, various headaches, many gastrointestinal symptoms, psychological symptoms, psychiatric episode(s) and mental health issues including depression and anxiety, sleep disturbances, and circulatory/breathing complaints (e.g., dyspnoeic symptoms, tachycardia, excessive perspiration, and menstruation disorders) as manifestations of stress (2, 6-11).

Such health symptoms/complaints have important implications for these young adults as they impact on students' academic performance. Generally, the deterioration in students' health may affect learning ability, academic performance and goal achievement (12). For instance, in relation to sleep quality, in Brazil, 
'sleepier' students did not achieve as well as the others in terms of their final examinations (13). There have also been many implications due to sleep quality among young people undertaking academic and professional training (14). Similarly, in connection with headaches, migraine and tension headaches, sufferers showed a substantial decrease in school productivity (7).

It has been proposed that stress could be associated with such self-reported conditions. University students experience considerable stress due new socializations, being away from family home, personal expectations and peer competition, having to achieved good grades, or students' fear of failing and repeating their course (3). Certainly, the university period could be stressful for students trying to achieve success in their academic goals despite financial constraints (15). In addition, some disciplines, e.g. undergraduate medical courses could prove to be inherently "stressful", due to the need to learn copious amounts of new information, and the constant requirement to interact with patients and translate clinical knowledge into practice (3). Further, some aspects of university accommodation for residence hall students, e.g. conflict with a roommate, or factors related to teachers - conflict with faculty or staff member, were also significant predictors of stress (16).

The literature reveals gaps in the knowledge base. First, whilst studies have documented the prevalence of various health complaints, less research explored the relationships between such health complaints/self-reported symptoms and the stress levels that university students actually perceive. Secondly, even less studies related these two features (health complaints and perceived stress) in countries of the Mediterranean basin, e.g. Egypt. Thirdly, while most studies typically collected data from several universities across countries or within one country $(15$, 17), less research has examined the same question (associations between health complaints and perceived stress) across students enrolled at different faculties of one university. The study described in this paper bridged these gaps in knowledge and surveyed undergraduate students across 11 faculties at an Egyptian university in order to explore the links between health complaints and perceived stress.

The current survey examined the links between perceived stress and a range of self reported symptoms and health complaints of a representative sample of undergraduate students $(\mathrm{N}=3,271)$ across eleven faculties at Assiut University in Assiut, Egypt. The four objectives were to:

- describe the sample's general characteristics and undertake factor analysis of 22 self reported symptoms/health complaints in order to categorise them into appropriate symptom groups;

- assess the prevalence of 22 symptoms/health complaints and the number of symptoms/health complaints reported in the last 12 months;

- assess the association between the frequency of symptoms/ health complaints and students' general demographic characteristics and health behaviours;

- explore the frequency of symptoms/health complaints by perceived stress level, and the association between symptoms/ health complaints and perceived stress while controlling for all other symptom groups;

- examine differences in levels of psychological symptoms and of perceived stress across different faculties.

\section{MATERIALS AND METHODS}

\section{Design, Data Collection and Ethical Considerations}

After ethical approval by the University ethics committee, data were collected during the academic year 2009-2010 from a representative random sample of students $(\approx 10 \%$ of students) at each of the eleven participating faculties of Assiut University. These faculties comprised Business ( $\mathrm{N}=604,18.5 \%$ of the sample), Engineering ( $\mathrm{N}$ $=572,17.5 \%)$, Education $(\mathrm{N}=461,14.1 \%)$, Arts $(\mathrm{N}=424,13.0 \%)$, Social Work $(\mathrm{N}=328,10.0 \%)$, Sciences $(\mathrm{N}=202,6.3 \%)$, Physical Education ( $\mathrm{N}=178,5.4 \%)$, Computers \& Information $(\mathrm{N}=137$, $4.2 \%)$, Veterinary Medicine $(\mathrm{N}=131,4.0 \%)$, Specific Education $(\mathrm{N}=119,3.6 \%)$, and Agriculture $(\mathrm{N}=50,1.5 \%)$.

In line with other general student health and well-being studies $(15,17-23)$, no monetary or course credit incentives were provided for participation. Each questionnaire included a participant information sheet outlining the research aims/objectives. Data were confidential and protected, and students were informed that by completing the questionnaire, they consent to participate in the study. For quality assurance purposes, one person at one site entered all the data thus minimising potential data entry errors. Based on the number of questionnaires returned by students, the response rate was $\approx 90 \%$.

\section{Health and Well-being Measures}

The questionnaire included general health and well-being information, the Perceived Stress Scale, and 22 symptoms/health complaints. The information also comprised gender, age, marital status, year of study, living arrangements (during semester), smoking, alcohol consumption, subjective health status, health awareness, height and weight (to compute BMI), importance of religion/personal faith (religiosity), and income sufficiency.

Perceived Stress Scale (4 Items): Cohen's Perceived Stress Scale (PSS) in its 4 item short form assessed the extent to which participants considered life situations to be stressful (24). The PSS-4 assesses the degree to which situations in one's life over the past month are appraised as stressful. The items detect how unpredictable, uncontrollable, and overloaded respondents find their lives. All items began with the same phrase: "In the past month, how often have you felt...?" (5-point scale: $0=$ 'never', 1 = 'almost never', 2 = 'sometimes', 3 = 'fairly often', 4 = 'very often'). Higher scores indicated more perceived stress. In our sample, Cronbach's alpha of the PSS was 0.46.

Health problems, symptoms/health complaints (22 items): students rated 22 symptoms measuring a range of health complaints $(6,25,26)$. Sample items included stomach trouble/heartburn, back pain, rapid heart beats/circulatory problem/dizziness, headaches, sleep disorder/insomnia, concentration difficulties, neck and shoulder pain, and depressive mood. Respondents rated the question: "How often have you had these complaints during the past 12 months?" (4-point response scale, 1 = 'never', 4 = 'very often'). In our sample, Cronbach's alpha of the whole scale (22 items) was 0.87.

Marital status (1 item): "What is your marital status?" ('Married - civil marriage', 'Married - religious marriage', and 'Single').

Living arrangements (1 item): "Where do you live (during university/college term time)?" with five options ('I live alone', 'I live with my parents', 'I live at university hostel', 'i live with 
room-mate/s', other), later recoded into two options based on whether the participant was living with parents or not.

Tobacco smoking (1 item): "Within the last three months, how often did you smoke? (cigarettes, pipes, cigarillos, cigars)", rated on a 3-point response scale: 'daily', 'occasionally', and 'never' (26).

Illicit drug/s use (1 item): "Have you ever use/used drugs?" ('yes, regularly', 'yes, but only a few times', 'never'). The three options were recoded into two options based on whether the participant ever used illicit drug/s or not.

Frequency of alcohol consumption (1 item): "Over the past three months how often have you drunk alcohol, for example, beer?" (six response options: 'never', 'once a week or less', 'once a week', 'a few times each week', 'every day', and, 'a few times each day').

Subjective health status (1 item): "How would you rate your health in general?" (5-point scale response format: $1=$ 'excellent', 2 = 'very good', 3 = 'good', 4 = 'fair', 5 = 'poor') $(9,27)$.

Watch one's health (Health awareness) (1 item): "To what extent do you keep an eye on your health?" (4-point response scale: 1 = 'not at all' and 4 = 'very much') (6).

$B M I$ (reported): was calculated from self-reported weight and height using Metric BMI Formula: BMI $\left(\mathrm{kg} / \mathrm{m}^{2}\right)=$ weight in kilograms/the squared height $\left(\mathrm{m}^{2}\right)$. Based on the World Health Organization guidelines (28), BMI was categorised into: underweight $\left(\mathrm{BMI}<18.5 \mathrm{~kg} / \mathrm{m}^{2}\right)$, normal $\left(18.5 \leq \mathrm{BMI} \leq 24.9 \mathrm{~kg} / \mathrm{m}^{2}\right)$, overweight $\left(25.0 \leq \mathrm{BMI} \leq 29.9 \mathrm{~kg} / \mathrm{m}^{2}\right)$, or obese $(\mathrm{BMI} \geq 30.0$ $\left.\mathrm{kg} / \mathrm{m}^{2}\right)$.

$B M I$ (measured): was calculated from measured weight and height using the same Metric BMI Formula and cut-off levels as with the reported BMI. Measurements were undertaken using (Seca Digital Weight \& Height Scale), with height measured to the nearest $0.1 \mathrm{~cm}$ (barefooted), and body weight measured to the nearest $0.1 \mathrm{~kg}$ (light clothing and no footwear) (29).

Importance of religion/personal faith (religiosity - 1 item): "My religion is very important for my life?" (5-point response options: $1=$ 'strongly disagree' to $5=$ 'strongly agree'), later recoded into two options based on whether the participant was in agreement or not.

Income sufficiency (subjective economic situation) (1 item): students were asked how sufficient they considered the amount of money they have at their disposal (4-point scale: 'always sufficient', 'mostly sufficient', 'mostly insufficient', 'always insufficient').

Quality of one's life (1 item): "If you consider the quality of your life: How did things go for you in the last four weeks?" (5 response categories, $1=$ 'very badly' to $5=$ 'very well'). For the current analysis, this variable was recoded into three categories (30).

\section{Data Analysis}

SPSS v19.0 was used for the statistical analyses. Factor analysis (using principal component analysis - PCA) determined the number and composition of underlying dimensions to be used in subsequent analyses, by merging the initial 22 questionnaire items (symptoms/health complaints) into constructs (31). The data was suitable for factor analysis: the sample size $(3,271)$ was more than the minimum 300 recommended for PCA; the ratio of questionnaires to items was much higher than the suggested 10 to 1 ratio; and the correlation matrices for the factors revealed many significant coefficients that ranged from 0.3-0.55 (data not presented) $(32,33)$. A scree plot of eigenvalues determined the number of components, which is superior to retaining all the dimensions with eigenvalues $>1(34,35)$. In factor analysis and PCA, each component is constructed as a weighted average of the questions that comprise it (factor scores), i.e. each component can be regarded as scale measure of the collective severity of those questions that comprise the component (36). Hence, a component value close to 1 corresponds to 'never' (or no severity), whereas a component value close to 4 corresponds to 'very often'.

For internal consistency, reliability analyses (Cronbach's alpha) examined the items comprising each of the four components (groups of symptoms) that emerged from the factor analysis. Pearson's coefficient assessed the correlation between the four components that emerged.

For the subsequent analysis, we calculated a sum score for each of the four components (dimensions) of symptoms that emerged from the factor analysis, which was then divided by the number of items in each dimension. The scores for each dimension of symptoms were presented as means and standard deviations. For logistic regression analysis, the scores were dichotomized by median split.

For the descriptive analyses, categorical data were expressed as frequencies and percentages, and score data were presented as means and standard deviations. Perceived stress was categorized into four levels based on quartiles. The first quartile (Q1) represented the lowest quarter of perceived stress level; the fourth quartile $(\mathrm{Q} 4)$ represented the highest quarter of perceived stress. For each group of symptoms, we used two-sample t-test or ANOVA to compare the severity of symptoms across (e.g. year of study, age, marital status etc.). We used Bonferroni adjustment for multiple testing for the statistical comparisons presented in Tables 5 and 6.

Multiple binomial or multinomial logistic regression analyses examined the relationship between each of the four symptom groups and other students' characteristics adjusted for the effects of all other symptom groups. Variables only significant in initial bivariate tests were included in the final table. Both perceived stress and level of psychological symptoms were each categorised into three intensity levels ('low', 'medium' and 'high') using multiple comparisons (with $\alpha=0.05$ ) to detect homogenous sub-sets.

\section{RESULTS}

\section{General Characteristics}

Females comprised about half the sample (52.4\%) (Table 1). However, the proportions of females differed by faculty: Business (69\%), Engineering (70\%), Education (22\%), Arts (26\%), Social Work (35\%), Sciences (44\%), Physical Education (85\%), Computers \& Information (38\%), Veterinary Medicine (29\%), Specific Education (19\%), and Agriculture (44\%). Females were more represented at the three largest participating faculties (Business, Engineering, Physical Education, $\approx 42 \%$ of the participants). The resultant effect was that across the whole sample (university), the percentages of males and females were nearly equal (47.6\% and $52.4 \%$, respectively). 
Table 1. General characteristics of the sample

\begin{tabular}{|c|c|c|}
\hline & Characteristic & $\mathrm{N}(\%)$ \\
\hline \multirow[t]{3}{*}{ Gender } & Female & $1,698(52.4)$ \\
\hline & Male & $1,540(47.6)$ \\
\hline & Total & $3,238(100)$ \\
\hline \multirow[t]{4}{*}{ Age (years) } & $<20$ & $2,800(87)$ \\
\hline & $20-24$ & $407(12.6)$ \\
\hline & $\geq 25$ & $11(0.3)$ \\
\hline & Total & $3,218(100)$ \\
\hline \multirow[t]{4}{*}{ Marital status } & Married (civil marriage) & $31(1.0)$ \\
\hline & Married (religious marriage) & $15(0.5)$ \\
\hline & Single & $3,145(98.6)$ \\
\hline & Total & $3,191(100)$ \\
\hline \multirow[t]{5}{*}{ Year of study } & $1 \mathrm{st}$ & $22(0.7)$ \\
\hline & 2nd & $1,058(32.9)$ \\
\hline & $3 r d$ & $918(28.5)$ \\
\hline & $\geq 4$ th & $1,219(37.9)$ \\
\hline & Total & $3,217(100)$ \\
\hline \multirow[t]{3}{*}{ Living with parent } & Yes & $1,218(38.1)$ \\
\hline & No & $1,978(61.9)$ \\
\hline & Total & $3,196(100)$ \\
\hline \multirow[t]{4}{*}{ Smoking } & Daily & $100(3.2)$ \\
\hline & Occasional & $174(5.6)$ \\
\hline & Never & $2,850(91.2)$ \\
\hline & Total & $3,124(100)$ \\
\hline \multirow[t]{3}{*}{ Ever use of illicit drug/s } & No & $2,687(95.5)$ \\
\hline & Yes & $126(4.5)$ \\
\hline & Total & $2,813(100)$ \\
\hline \multirow[t]{5}{*}{ Alcohol consumption frequency } & Never & $2,520(89.6)$ \\
\hline & $\leq$ Once a week & $185(6.6)$ \\
\hline & Several times a week & $84(3.0)$ \\
\hline & Several times a day/Every day & $23(0.8)$ \\
\hline & Total & $2,812(100)$ \\
\hline \multirow[t]{4}{*}{ Subjective health status } & Excellent/Very good & $597(18.4)$ \\
\hline & Good & $1,531(47.2)$ \\
\hline & Fair/Poor & $1,114(34.4)$ \\
\hline & Total & $3,242(100)$ \\
\hline \multirow[t]{3}{*}{ Watch one's health (health awareness) } & Very much/To some extent & $2,414(74.7)$ \\
\hline & Not much/Not at all & $817(25.3)$ \\
\hline & Total & $3,231(100)$ \\
\hline \multirow[t]{5}{*}{ BMI (reported) a } & Underweight & $124(7.6)$ \\
\hline & Normal weight & $1,039(64.1)$ \\
\hline & Overweight & $335(20.7)$ \\
\hline & Obese & $123(7.6)$ \\
\hline & Total & $1,621(100)$ \\
\hline
\end{tabular}


Cont. from page 71

\begin{tabular}{|l|l|l|}
\hline \multirow{4}{*}{ BMI (measured) } & Characteristic & $\mathbf{N}(\%)$ \\
\hline \multirow{5}{*}{ Importance of religion (religiosity) } & Underweight & $199(6.3)$ \\
\cline { 2 - 3 } & Normal weight & $2,056(64.7)$ \\
\cline { 2 - 3 } & Overweight & $709(22.3)$ \\
\cline { 2 - 3 } & Obese & $214(6.7)$ \\
\cline { 2 - 3 } & Total & $3,178(100)$ \\
\hline \multirow{5}{*}{ Income sufficiency } & Somewhat/Strongly disagree & $42(1.3)$ \\
\cline { 2 - 3 } & Strongly/Somewhat agree & $3,200(98.7)$ \\
\cline { 2 - 3 } & Total & $3,242(100)$ \\
\hline \multirow{5}{*}{ Quality of life } & Always/Mostly sufficient & $2,460(77.6)$ \\
\cline { 2 - 3 } & Mostly/Always insufficient & $712(22.5)$ \\
\cline { 2 - 3 } & Total & $3,172(100)$ \\
\hline & Very badly/Badly & $427(13.2)$ \\
\cline { 2 - 3 } & Intermediate & $1,566(48.5)$ \\
\cline { 2 - 3 } & Quite well/Very well & $1,235(38.3)$ \\
\cline { 2 - 3 } & Total & $3,228(100)$ \\
\hline
\end{tabular}

${ }^{a}$ Calculated based on self- reported height and weight and, ${ }^{\mathrm{b}}$ Calculated based on objectively measured height and weight, both categorised according to the $\mathrm{WHO}$ guidelines - underweight $\left(\mathrm{BMI}<18.5 \mathrm{~kg} / \mathrm{m}^{2}\right)$, normal weight (BMl of $\left.18.5-24.9 \mathrm{~kg} / \mathrm{m}^{2}\right)$, overweight (BMl of $\left.25.0-29.9 \mathrm{~kg} / \mathrm{m}^{2}\right)$, or obese $\left(\mathrm{BMI} \geq 30.0 \mathrm{~kg} / \mathrm{m}^{2}\right)(28)$.

Table 2. Factor analysis of 22 self reported symptoms/health complaints into four components

\begin{tabular}{|c|c|c|c|c|}
\hline & \multicolumn{4}{|c|}{ Component } \\
\hline & 1 & 2 & 3 & 4 \\
\hline & $\begin{array}{l}\text { Psychological } \\
\text { (9 items) }\end{array}$ & $\begin{array}{c}\text { Circulatory/Breathing } \\
\text { (5 items) }\end{array}$ & $\begin{array}{l}\text { Gastrointestinal } \\
\quad(3 \text { items })\end{array}$ & $\begin{array}{l}\text { Pains/Aches } \\
\text { (5 items) }\end{array}$ \\
\hline Cronbach's alpha & 0.80 & 0.72 & 0.69 & 0.68 \\
\hline Nervousness/Anxiety & 0.712 & & & \\
\hline Depressive mood & 0.661 & & & \\
\hline Mood swings & 0.605 & & & \\
\hline Fear/Phobia & 0.593 & & & \\
\hline Difficulties to concentrate & 0.568 & & & \\
\hline Sleep disorder/Insomnia & 0.565 & & & \\
\hline Nightmares & 0.532 & & & \\
\hline Lack of appetite & 0.497 & & & \\
\hline Weight gain/Weight loss & 0.462 & & & \\
\hline Trembling hands & & 0.753 & & \\
\hline Trembling & & 0.749 & & \\
\hline Speech impediment & & 0.610 & & \\
\hline Breathing difficulties & & 0.509 & & \\
\hline Rapid heartbeats/Circulatory problems/Dizziness & & 0.443 & & \\
\hline Diarrhoea & & & 0.821 & \\
\hline Constipation & & & 0.816 & \\
\hline Abdominal problems & & & 0.558 & \\
\hline Back pain & & & & 0.704 \\
\hline Neck and shoulder pain & & & & 0.597 \\
\hline Fatigue & & & & 0.552 \\
\hline Stomach trouble/Heartburn & & & & 0.522 \\
\hline Headaches & & & & 0.442 \\
\hline
\end{tabular}


Table 3. Prevalence of symptoms/health complaints during last 12 months

\begin{tabular}{|c|c|c|c|}
\hline & $\begin{array}{l}\text { Never } \\
\mathrm{N}(\%)\end{array}$ & $\begin{array}{l}\text { Rarely } \\
\mathrm{N}(\%)\end{array}$ & $\begin{array}{c}\text { Sometimes/Very often } \\
\mathrm{N}(\%)\end{array}$ \\
\hline \multicolumn{4}{|l|}{ Psychological } \\
\hline Depressive mood & $635(20.2)$ & $778(24.7)$ & $1,732(55.1)$ \\
\hline Nervousness/Anxiety & $474(15.0)$ & $692(21.9)$ & $1,999(63.2)$ \\
\hline Mood swings & $308(9.8)$ & $498(15.8)$ & $2,350(74.5)$ \\
\hline Difficulties to concentrate & $155(4.9)$ & $539(17.0)$ & $2,478(78.1)$ \\
\hline Fear/Phobia & $663(22.2)$ & $754(25.2)$ & $1,572(52.6)$ \\
\hline Nightmares & $786(25.1)$ & $1,053(33.6)$ & $1,298(41.4)$ \\
\hline Weight gain/Weight loss & $632(20.2)$ & $771(24.6)$ & $1,728(55.2)$ \\
\hline Lack of appetite & $803(25.5)$ & $913(28.9)$ & $1,438(45.6)$ \\
\hline Sleep disorder/Insomnia & $465(14.8)$ & $677(21.5)$ & $2,008(63.7)$ \\
\hline \multicolumn{4}{|l|}{ Circulatory/Breathing } \\
\hline Trembling hands & $1,597(51.2)$ & $817(26.2)$ & $707(22.7)$ \\
\hline Trembling & $1,553(50.0)$ & $920(29.6)$ & $632(20.4)$ \\
\hline Speech impediment & $2,013(64.9)$ & $782(19.7)$ & $479(15.4)$ \\
\hline Rapid heartbeats, circulatory problems, dizziness & $988(31.5)$ & $745(24.9)$ & $1,370(43.6)$ \\
\hline Breathing difficulties & $1,417(45.1)$ & $982(31.3)$ & $741(23.6)$ \\
\hline \multicolumn{4}{|l|}{ Gastrointestinal } \\
\hline Diarrhoea & $1,062(34)$ & $1,210(38.7)$ & $856(27.4)$ \\
\hline Constipation & $1,091(34.9)$ & $1,129(36.2)$ & $902(28.9)$ \\
\hline Abdominal problems & $444(14.3)$ & $918(29.6)$ & $1,735(56)$ \\
\hline \multicolumn{4}{|l|}{ Pains/Aches } \\
\hline Back pain & $678(21.5)$ & $748(23.7)$ & $1,724(54.7)$ \\
\hline Neck and shoulder pain & $635(20.2)$ & $878(27.9)$ & $1,630(51.9)$ \\
\hline Fatigue & $160(5.1)$ & $304(9.6)$ & $2,703(85.3)$ \\
\hline Stomach trouble/Heartburn & $884(28.5)$ & $760(24.5)$ & $1,459(47.0)$ \\
\hline Headaches & $196(6.2)$ & $506(16)$ & $2,470(77.9)$ \\
\hline
\end{tabular}

All percentages are row percentages and rounded to one decimal point

Table 4. Number of symptoms/health complaints reported in last 12 months

\begin{tabular}{|l|c|c|c|c|}
\hline & Items & $\begin{array}{c}\text { No Symptoms } \\
\mathbf{N}(\%)\end{array}$ & $\begin{array}{c}\text { 1-2 Symptoms } \\
\mathbf{N}(\%)\end{array}$ & $\begin{array}{c}\geq 3 \text { Symptoms } \\
\mathbf{N}(\%)\end{array}$ \\
\hline Psychological & 9 & $95(3.5)$ & $298(10.9)$ & $2,349(85.7)$ \\
\hline Circulatory/Breathing & 5 & $1,098(37.2)$ & $1,345(45.5)$ & $512(17.3)$ \\
\hline Gastrointestinal & 3 & $1,037(34.6)$ & $1,528(50.9)$ & $436(14.5)$ \\
\hline Pains/Aches & 4 & $127(4.3)$ & $802(27.0)$ & $2,043(68.7)$ \\
\hline
\end{tabular}

All symptoms counted if reported to occur sometimes or very often

Most students $(87.0 \%)$ were $<20$ years old and in the 1 st to 3rd year of study $(62.1 \%)$. While $98.6 \%$ of students were single, the majority did not live with their parents (61.9\%). Only $2.5 \%$ felt their income was mostly/always sufficient, and most students $(98.7 \%)$ viewed religiosity as important. Two thirds of the participants reported good to excellent health $(65.6 \%)$, and most students 'kept an eye' on their health $(74.4 \%)$, but only $38.3 \%$ of students reported quite well/very well quality of life. Based on BMI, $\approx 29 \%$ were overweight or obese. Only $8.8 \%$ smoked at least occasionally, $4.5 \%$ had ever tried illicit drug/s, and most students $(89.6 \%)$ never consumed alcohol.
Data Reduction: Four Groups (Components) of Symptoms/Health Complaints

The factor analysis grouped the 22 questionnaire items into manageable dimensions. Four groups of symptoms (components) emerged (Table 2). Their corresponding $\alpha$ were all within $0.68-0.80$. The first group comprised psychological complaints (9 symptoms - Nervousness/Anxiety, Depressive mood, Mood swings, Fear/Phobia, Difficulties to concentrate, Sleep disorder/ Insomnia, Nightmares, Lack of appetite, Weight gain/loss). The second group represented circulatory/breathing complaints (5 symptoms - Trembling hands, Trembling, Speech impediment, 
Table 5. Frequency of symptoms/health complaints by general characteristics and by health behaviour

\begin{tabular}{|c|c|c|c|c|c|c|c|c|}
\hline & $\begin{array}{c}\text { Psychologi- } \\
\text { cal } \\
\text { M (SD) }\end{array}$ & $\mathbf{P}$ & $\begin{array}{c}\text { Circulatory/ } \\
\text { Breathing } \\
\text { M (SD) }\end{array}$ & $\mathbf{P}$ & $\begin{array}{c}\text { Gastrointes- } \\
\text { tinal } \\
\text { M (SD) }\end{array}$ & $\mathbf{P}$ & $\begin{array}{c}\text { Pains/Aches } \\
\text { M (SD) }\end{array}$ & $\mathbf{P}$ \\
\hline \multicolumn{9}{|l|}{ Gender } \\
\hline Female & $2.77(0.6)$ & \multirow{2}{*}{$<0.001$} & $1.91(0.6)$ & \multirow{2}{*}{$<0.001$} & $2.29(0.7)$ & \multirow{2}{*}{$<0.001$} & $2.80(0.6)$ & \multirow{2}{*}{$<0.001$} \\
\hline Male & $2.48(0.6)$ & & $1.76(0.6)$ & & $2.03(0.7)$ & & $2.59(0.7)$ & \\
\hline \multicolumn{9}{|l|}{ Age } \\
\hline$<20$ & $2.64(0.6)$ & \multirow{3}{*}{0.12} & $1.84(0.5)$ & \multirow{3}{*}{0.35} & $2.17(0.7)$ & \multirow{3}{*}{0.65} & $2.70(0.6)$ & \multirow{3}{*}{0.49} \\
\hline $20-24$ & $2.58(0.6)$ & & $1.85(0.5)$ & & $2.16(0.7)$ & & $2.68(0.6)$ & \\
\hline$\geq 24$ & $2.57(0.8)$ & & $2.11(0.5)$ & & $2.00(1.0)$ & & $2.89(0.9)$ & \\
\hline \multicolumn{9}{|l|}{ Marital status } \\
\hline Married (religious marriage) & $2.71(0.6)$ & \multirow{3}{*}{0.29} & $2.10(0.7)$ & \multirow{3}{*}{0.05} & $2.26(0.9)$ & \multirow{3}{*}{0.47} & $2.85(0.5)$ & \multirow{3}{*}{0.08} \\
\hline Married (civil marriage) & $2.41(0.8)$ & & $1.67(0.8)$ & & $1.97(0.7)$ & & $2.42(0.8)$ & \\
\hline Single & $2.63(0.6)$ & & $1.84(0.6)$ & & $2.20(0.7)$ & & $2.70(0.6)$ & \\
\hline \multicolumn{9}{|l|}{ Year of study } \\
\hline 1st & $2.51(0.6)$ & \multirow{4}{*}{$<0.001$} & $1.74(0.7)$ & \multirow{4}{*}{0.86} & $2.13(0.7)$ & \multirow{4}{*}{0.002} & $2.64(0.6)$ & \multirow{4}{*}{$<0.001$} \\
\hline 2nd & $2.60(0.6)$ & & $1.84(0.7)$ & & $2.10(0.7)$ & & $2.62(0.6)$ & \\
\hline 3rd & $2.71(0.6)$ & & $1.85(0.6)$ & & $2.20(0.7)$ & & $2.80(0.6)$ & \\
\hline$\geq 4$ th & $2.61(0.6)$ & & $1.84(0.6)$ & & $2.21(0.7)$ & & $2.70(0.6)$ & \\
\hline \multicolumn{9}{|l|}{ Living with parent } \\
\hline Yes & $2.60(0.6)$ & \multirow{2}{*}{$<0.001$} & $1.78(0.6)$ & \multirow{2}{*}{$<0.001$} & $2.11(0.7)$ & & $2.70(0.6)$ & \\
\hline No & $2.68(0.6)$ & & $1.88(0.7)$ & & $2.20(0.7)$ & -0.001 & $2.72(0.6)$ & $0.0<0$ \\
\hline Smoking & & & & & & & & \\
\hline Daily & $2.67(0.6)$ & & $1.99(0.7)$ & & $1.07(0.8)$ & & $2.69(0.7)$ & \\
\hline Occasional & $2.52(0.6)$ & 0.038 & $1.81(0.6)$ & 0.057 & $2.03(0.7)$ & 0.006 & $2.63(0.7)$ & 0.30 \\
\hline Never & $2.64(0.6)$ & & $1.84(0.6)$ & & $2.18(0.7)$ & & $2.71(0.6)$ & \\
\hline Ever use of illicit drug/s & & & & & & & & \\
\hline No & $2.63(0.6)$ & (207 & $1.84(0.7)$ & م0 & $1.17(0.7)$ & 0005 & $2.70(0.6)$ & \\
\hline Yes & $2.64(0.6)$ & 0.01 & $1.96(0.7)$ & 0.04 & $1.99(0.7)$ & 0.005 & $2.68(0.7)$ & 0.04 \\
\hline Alcohol consumption frequenc & & & & & & & & \\
\hline Never & $2.64(0.6)$ & & $1.84(0.7)$ & & $2.17(0.7)$ & & $2.70(0.6)$ & \\
\hline$\leq$ Once a week & $2.55(0.6)$ & 07 & $1.86(0.7)$ & 075 & $2.15(0.7)$ & 073 & $2.63(0.6)$ & 0.54 \\
\hline Several times a week & $2.58(0.6)$ & 0.01 & $1.77(0.6)$ & 0.10 & $2.12(0.7)$ & 0.10 & $2.68(0.6)$ & 0.04 \\
\hline Several times a day/Every day & $2.84(0.6)$ & & $1.80(0.6)$ & & $2.04(0.8)$ & & $2.69(0.6)$ & \\
\hline Subjective health & & & & & & & & \\
\hline Excellent/Very good & $2.35(0.6)$ & & $1.54(0.4)$ & & $2.00(0.7)$ & & $2.41(0.7)$ & \\
\hline Good & $2.60(0.6)$ & $<0.001$ & $1.80(0.5)$ & $<0.001$ & $2.15(0.7)$ & $<0.001$ & $2.68(0.6)$ & $<0.001$ \\
\hline Fair/Poor & $2.84(0.7)$ & & $2.05(0.7)$ & & $2.28(0.7)$ & & $2.88(0.6)$ & \\
\hline Watch one's health (health aw & areness) & & & & & & & \\
\hline Very much/Some extent & $2.58(0.6)$ & 80001 & $1.79(0.6)$ & -0001 & $2.15(0.7)$ & 2002 & $2.66(0.6)$ & $8 \cap 001$ \\
\hline Not much/Not at all & $2.78(0.6)$ & $<0.001$ & $1.97(0.7)$ & $<.001$ & $2.23(0.7)$ & 0.002 & $2.82(0.6)$ & $<0.001$ \\
\hline BMI (reported) & & & & & & & & \\
\hline Underweight & $2.74(0.6)$ & & $1.89(0.6)$ & & $2.11(0.6)$ & & $2.71(0.7)$ & \\
\hline Normal weight & $2.58(0.6)$ & 0024 & $1.81(0.7)$ & 052 & $2.11(0.7)$ & 0077 & $2.65(0.6)$ & 0010 \\
\hline Overweight & $2.62(0.6)$ & $0.0 \angle 4$ & $1.79(0.6)$ & 0.00 & $2.20(0.7)$ & 0.001 & $2.75(0.6)$ & 0.019 \\
\hline Obese & $2.67(0.6)$ & & $1.83(0.7)$ & & $2.25(0.7)$ & & $2.78(0.6)$ & \\
\hline
\end{tabular}


Cont. from page 74

\begin{tabular}{|c|c|c|c|c|c|c|c|c|}
\hline & $\begin{array}{c}\text { Psychologi- } \\
\text { cal } \\
\text { M (SD) }\end{array}$ & $\mathbf{P}$ & $\begin{array}{c}\text { Circulatory/ } \\
\text { Breathing } \\
M(S D)\end{array}$ & $\mathbf{P}$ & $\begin{array}{c}\text { Gastrointes- } \\
\text { tinal } \\
\text { M (SD) }\end{array}$ & $\mathbf{P}$ & $\begin{array}{c}\text { Pains/Aches } \\
\text { M (SD) }\end{array}$ & $\mathbf{P}$ \\
\hline \multicolumn{9}{|l|}{ BMI (measured) } \\
\hline Underweight & $2.72(0.7)$ & \multirow{4}{*}{0.045} & $1.83(0.6)$ & \multirow{4}{*}{0.93} & $2.10(0.7)$ & \multirow{4}{*}{0.01} & $2.67(0.6)$ & \multirow{4}{*}{0.019} \\
\hline Normal weight & $2.61(0.6)$ & & $1.85(0.7)$ & & $2.15(0.7)$ & & $2.68(0.6)$ & \\
\hline Overweight & $2.67(0.6)$ & & $1.83(0.6)$ & & $2.23(0.7)$ & & $2.76(0.6)$ & \\
\hline Obese & $2.65(0.6)$ & & $1.85(0.6)$ & & $2.25(0.7)$ & & $2.74(0.6)$ & \\
\hline \multicolumn{9}{|c|}{ Importance of religion (religiosity) } \\
\hline Somewhat/Strongly disagree & $2.49(0.6)$ & \multirow{2}{*}{0.13} & $1.70(0.6)$ & \multirow{2}{*}{0.15} & $2.04(0.6)$ & \multirow{2}{*}{0.14} & $2.59(0.6)$ & \multirow{2}{*}{0.24} \\
\hline Strongly/Somewhat agree & $2.64(0.6)$ & & $1.84(0.6)$ & & $2.17(0.7)$ & & $2.70(0.6)$ & \\
\hline \multicolumn{9}{|l|}{ Income sufficiency } \\
\hline Always/Mostly sufficient & $2.60(0.6)$ & \multirow{2}{*}{$<0.001$} & $1.79(0.6)$ & \multirow{2}{*}{$<0.001$} & $2.15(0.7)$ & \multirow{2}{*}{0.001} & $2.67(0.6)$ & \multirow{2}{*}{$<0.001$} \\
\hline Mostly/Always insufficient & $2.74(0.6)$ & & $2.00(0.7)$ & & $2.25(0.7)$ & & $2.80(0.6)$ & \\
\hline \multicolumn{9}{|l|}{ Quality of life } \\
\hline Very badly/Badly & $3.00(0.6)$ & \multirow{3}{*}{$<0.001$} & $2.11(0.7)$ & \multirow{3}{*}{$<0.001$} & $2.30(0.7)$ & \multirow{3}{*}{$<0.001$} & $2.92(0.6)$ & \multirow{3}{*}{$<0.001$} \\
\hline Intermediate & $2.68(0.6)$ & & $1.87(0.6)$ & & $1.19(0.7)$ & & $2.74(0.6)$ & \\
\hline Quite well/Very well & $2.46(0.6)$ & & $1.70(0.6)$ & & $2.11(0.7)$ & & $2.59(0.6)$ & \\
\hline
\end{tabular}

M: mean; SD: standard deviation rounded to one decimal point; all p-values based on t-test or ANOVA; significance level after Bonferoni adjustment set at $p<0.001$; symptoms measured on a four-point response scale, 1 = 'never'; 4 = 'very often'

Table 6. Frequency of symptoms/health complaints by level of perceived stress

\begin{tabular}{|l|c|c|c|c|c|}
\hline \multirow{2}{*}{} & \multicolumn{4}{|c|}{ Level of perceived stress } & \multirow{2}{*}{ Q4 } \\
\cline { 2 - 6 } & Q1 & Q2 & Q3 & M \\
\cline { 2 - 6 } & M (SD) & M (SD) & M (SD) & M & \\
\hline Psychological symptoms & $2.45(0.6)$ & $2.60(0.6)$ & $2.76(0.6)$ & $2.94(0.5)$ & $<0.001$ \\
\hline Circulatory/Breathing symptoms & $1.72(0.6)$ & $1.83(0.6)$ & $1.89(0.7)$ & $2.03(0.7)$ & $<0.001$ \\
\hline Gastrointestinal symptoms & $2.11(0.7)$ & $2.13(0.7)$ & $2.22(0.7)$ & $2.31(0.7)$ & $<0.001$ \\
\hline Pains/Aches symptoms & $2.59(0.6)$ & $2.67(0.6)$ & $2.79(0.6)$ & $2.89(0.6)$ & $<0.001$ \\
\hline
\end{tabular}

Q: quartile (Q4 = highest level of perceived stress); M: mean; SD: standard deviation rounded to one decimal point; symptoms measured on four-point response scale ( 1 = 'never'; 4 = 'very often'); $p$-values based on ANOVA tests.

Breathing difficulties, and Rapid heartbeats, Circulatory problems and Dizziness). The third group encompassed gastrointestinal complaints (3 symptoms - Diarrhoea, Constipation, Abdominal problems). Finally, the fourth group embraced some pains/aches (5 symptoms - Back pain, Neck and shoulder pain, Fatigue, Stomach trouble/Heartburn, Headaches). Generally, for most items, their corresponding factor loadings were $\geq 0.5$ and reaching 0.821 , with few exceptions where loadings were between 0.442 and 0.497 .

\section{Prevalence and Number of Symptoms/Health Com- plaints Reported in the Last 12 Months}

The questionnaire comprised 22 symptoms. For the psychological symptoms, participants reported that difficulties to concentrate $(78.1 \%)$ and mood swings (74.5\%) occurred sometimes/very often during the last 12 months (Table 3 ). Similarly, nervousness/ anxiety $(63.2 \%)$ and sleep disorder $(63.7 \%)$ affected many students. In relation to the pains/aches group, participants reported fatigue $(85.3 \%)$ and headache $(77.9 \%)$. As for circulatory/breathing symptoms or the group of gastrointestinal symptoms, relatively fewer students $(<30 \%$ of students) reported anything, with the exception of abdominal problems (56\%) and circulatory problems (43.6\%). The majority of students had up to two symptoms of the circulatory/breathing group and of the gastrointestinal symptoms group sometimes/very often during the last 12 months (Table 4). About $85.7 \%$ of the sample reported $\geq 3$ psychological symptoms and $68.7 \%$ reported $\geq 3$ pains/aches symptoms.

\section{Frequency of Symptoms by General Characteristics}

The frequency of symptoms in the four symptom groups expressed as mean rating (from $1=$ 'never' to $4=$ 'very often') by students' general characteristics and health behaviours are shown in Table 5. After Bonferroni adjustment, some associations were significant: across all four symptom groups, females had higher ratings of complaints than males; and students with insufficient income had higher ratings than students who were more satisfied with their income.

Participants perceiving their health as fair/poor had consistently higher ratings across all symptom groups when compared with those with very good/excellent health. The same trend was observed in relation to quality of life, where students reporting bad/very bad quality of life showed consistently higher ratings of symptoms. 
Table 7. Adjusted odds ratio for symptoms/health complaints by levels of perceived stress and by general characteristics

\begin{tabular}{|c|c|c|c|c|}
\hline \multirow{2}{*}{ Variable } & Psychological & Circulatory/Breathing & Gastrointestinal & Pains/Aches \\
\hline & OR $(95 \% \mathrm{Cl})$ & OR $(95 \% \mathrm{Cl})$ & OR $(95 \% \mathrm{Cl})$ & OR $(95 \% \mathrm{Cl})$ \\
\hline \multicolumn{5}{|l|}{ Perceived stress } \\
\hline Q1 & 1.00 & 1.00 & 1.00 & 1.00 \\
\hline Q2 & $1.61(1.32-1.96)$ & $1.15(0.69-1.37)$ & $0.88(0.76-1.02)$ & $0.94(0.78-1.13)$ \\
\hline Q3 & $2.54(2.04-3.17)$ & $0.97(0.80-1.17)$ & $0.91(0.76-1.07)$ & $1.15(0.94-1.42)$ \\
\hline Q4 & $4.51(3.50-5.8)$ & $1.04(0.85-1.28)$ & $0.93(0.78-1.11)$ & $1.15(0.91-1.45)$ \\
\hline \multicolumn{5}{|c|}{ General characteristics } \\
\hline \multicolumn{5}{|l|}{ Gender } \\
\hline Male & 1.00 & 1.00 & 1.00 & 1.00 \\
\hline Female & $2.19(1.86-2.58)$ & $0.81(0.71-0.94)$ & $1.39(1.23-1.56)$ & $1.16(0.99-1.35)$ \\
\hline \multicolumn{5}{|l|}{ Year of study } \\
\hline $1 s t^{*}$ & 1.00 & 1.00 & 1.00 & 1.00 \\
\hline 2nd & $1.39(0.54-3.53)$ & $1.33(0.55-3.18)$ & $0.85(0.42-1.72)$ & $0.75(0.30-1.83)$ \\
\hline $3 \mathrm{rd}$ & $1.52(0.59-3.90)$ & $1.00(0.42-2.41)$ & $0.91(0.45-1.85)$ & $1.26(0.51-3.10)$ \\
\hline$\geq 4$ th & $1.15(0.45-2.94)$ & $1.17(0.49-2.81)$ & $1.05(0.52-2.12)$ & $0.99(0.41-2.43)$ \\
\hline \multicolumn{5}{|l|}{ Living with parent } \\
\hline No & 1.00 & 1.00 & 1.00 & 1.00 \\
\hline Yes & $0.70(0.60-0.82)$ & $0.93(0.81-1.07)$ & $0.91(0.81-1.02)$ & $1.13(0.97-1.32)$ \\
\hline \multicolumn{5}{|l|}{ Subjective health status } \\
\hline Excellent/Very good & 1.00 & 1.00 & 1.00 & 1.00 \\
\hline Good & $1.37(1.11-1.67)$ & $1.56(1.26-1.93)$ & $0.93(0.79-1.09)$ & $1.54(1.26-1.88)$ \\
\hline Fair/Poor & $2.13(1.69-2.69)$ & $2.56(1.80-2.82)$ & $0.91(0.77-1.09)$ & $1.91(1.53-2.38)$ \\
\hline \multicolumn{5}{|l|}{ Watch one's health } \\
\hline Not much/Not at all & 1.00 & 1.00 & 1.00 & 1.00 \\
\hline Very much/Some extent & $0.68(0.57-0.81)$ & $0.82(0.71-0.96)$ & $1.07(0.94-1.22)$ & $0.85(0.72-1.00)$ \\
\hline \multicolumn{5}{|l|}{ Income sufficiency } \\
\hline Always/Mostly insufficient & 1.00 & 1.00 & 1.00 & 1.00 \\
\hline Always/Mostly sufficient & $0.89(0.74-1.07)$ & $0.69(0.59-0.82)$ & $0.99(0.86-1.14)$ & $0.88(0.74-1.05)$ \\
\hline \multicolumn{5}{|l|}{ Quality of life } \\
\hline Very badly/Badly & 1.00 & 1.00 & 1.00 & 1.00 \\
\hline Intermediate & $0.37(0.28-0.47)$ & $0.87(0.72-1.07)$ & $1.08(0.90-1.30)$ & $0.96(0.76-1.22)$ \\
\hline Quite well/Very well & $0.20(0.16-0.27)$ & $0.73(0.59-0.91)$ & $1.19(0.98-1.44)$ & $0.90(0.70-1.15)$ \\
\hline
\end{tabular}

Multinominal logistic regression models; OR: odds ratio (adjusted for the other three groups of symptoms); Cl: confidence interval; bolded cells indicate statistical significance.

Conversely, students who watched their health to some extent/very much had generally lower ratings of symptoms, although for gastrointestinal symptoms this did not reach statistical significance. Students exhibited increasing frequency of complaints with higher year of study for psychological and pains/aches symptoms. Participants not living with their parents had higher ratings of complaints for all groups except for pains/aches. The associations between symptoms and all the remaining variables did not reach statistical significance.

\section{Association between Perceived Stress and Frequency of Self Reported Symptoms/Health Complaints}

The frequency of symptoms across the four symptom groups expressed as mean rating (from $1=$ 'never' to $4=$ 'very often') by quartiles of perceived stress are depicted in Table 6. A significant association between the frequency of symptoms and the level of perceived stress is shown by ANOVA tests over all levels of perceived stress. There was a trend of increasing frequency of symptoms with increasing levels of perceived stress that was significant for all 4 groups of symptoms (all p-values $<0.001$ ) (Fig. 1). The graph shows the positive slope and the $95 \%$ confidence intervals (error bars) confirming the significant positive trend (approx. p-values $<0.002$ ).

We explored the associations between increasing perceived stress levels and higher frequency of the four symptom groups as dependent variables when adjusting for other groups of symptoms. After adjusting for the other groups of symptoms, increasing odds ratios with increasing level of perceived stress were only noted for psychological symptoms (Table 7). 


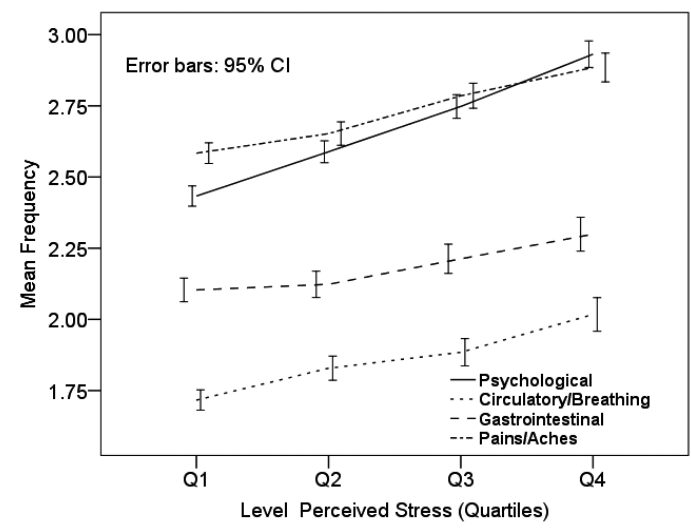

Fig. 1. Increasing frequency of symptoms/health complaints with increasing level of perceived stress.

However, across all the symptom groups, perceived poor health was consistently associated with higher frequency of symptoms. Similarly, students who watched their health (higher health awareness) were less likely to report psychological and circulatory/ breathing symptoms. Better quality of life was associated with lower frequency of only the psychological and circulatory/breathing symptoms, but not with the other two symptom groups. Females were more likely to report psychological and gastrointestinal symptoms, but less likely to feel circulatory/breathing symptoms. Students were less likely to report psychological symptoms if they lived with their parents and less likely to report circulatory/ breathing symptoms if their income was always/mostly sufficient.

\section{Differences in Levels of Psychological Symptoms and of Perceived Stress by Faculty}

The levels of psychological symptoms and of perceived stress differed significantly by faculty (ANOVA between groups for psychological symptoms $\mathrm{df}=10, \mathrm{~F}=10.1, \mathrm{p}<0.001$; ANOVA between groups for perceived stress $\mathrm{df}=10, \mathrm{~F}=9.5, \mathrm{p}<0.001$ ) (Fig. 2).

We then categorised the findings for each faculty into high, medium and low levels for both the psychological symptoms and perceived stress (Table 8). Students studying Physical Education were lowest in both psychological symptoms and perceived stress; students at Social Work faculty were low in psychological symptoms and medium in perceived stress; and Veterinary Medicine students were highest for both scores (Table 8). Students enrolled

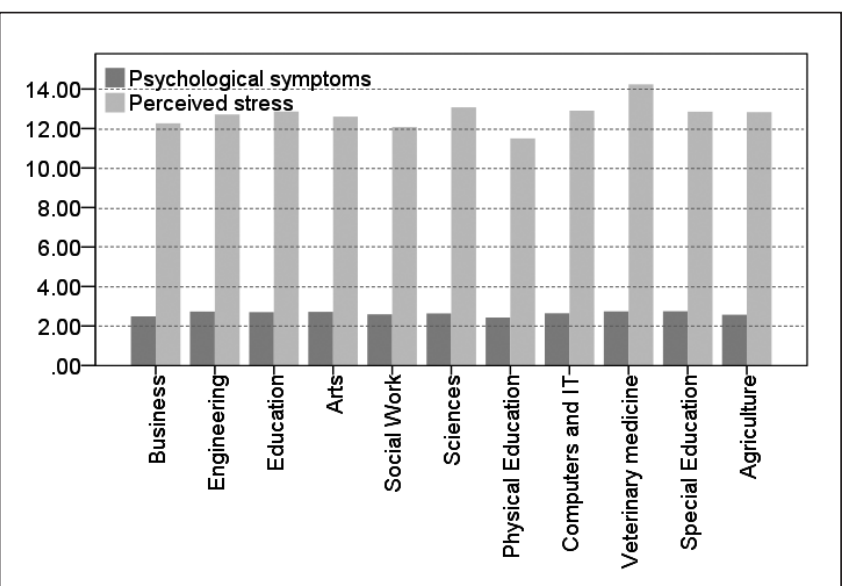

Fig. 2. Mean scores of psychological symptoms and level of perceived stress by faculty.

at Education, Arts, Engineering, and Special Education exhibited high mean scores in psychological symptoms, but only medium perceived stress.

\section{DISCUSSION}

This cross-sectional survey assessed the relationships between perceived stress and a range of self reported symptoms/health complaints among university students in Egypt.

As for objective one, four groups of symptoms emerged: psychological; circulatory/breathing; gastrointestinal; and pains/ aches. Few other studies investigated such a wide range of symptoms and factor analysed them into components. The HBSC Symptom Checklist used in the Health Behaviour in School-aged Children Study contained only eight symptoms and factor analysed them into two groups (37). Likewise, across a university student samples from Germany, Spain and Lithuania, a symptom checklist similar to that we employed (but containing only 9 of the symptoms) was categorised into three symptom groups: psychosomatic complaints (headache, nervousness, depression, sleep disturbance, dizziness); gastrointestinal complaints (diarrhea and constipation); and neck ache/backache (6). In contrast to our current findings, circulatory/breathing symptoms did not comprise a symptom group in the Stock et al. study (6). This was probably due to that the shorter (more brief) symptoms scale that they used containing only dizziness as circulatory/breathing symptoms, and

Table 8. Faculties by level of perceived stress and level of psychological symptoms

\begin{tabular}{|c|c|c|c|c|}
\hline \multirow{5}{*}{ 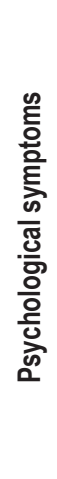 } & & \multicolumn{3}{|c|}{ Perceived stress } \\
\hline & & Low & Medium & High \\
\hline & Low & Physical Education & Business & \\
\hline & Medium & Social Work & $\begin{array}{l}\text { Agriculture } \\
\text { Social Work } \\
\text { Computers and IT } \\
\text { Sciences }\end{array}$ & \\
\hline & High & & $\begin{array}{l}\text { Education } \\
\text { Arts } \\
\text { Engineering } \\
\text { Special Education }\end{array}$ & Veterinary Medicine \\
\hline
\end{tabular}


other relevant symptoms were lacking in that scale (6). However, in agreement with our current findings, a 16-item list of symptoms comparable to those used in the current study was also similarly condensed into four parallel symptom groups in a sample of German university students (38). This indicated that the symptoms list functioned similarly in assessing self-reported health complaints across university students with western (European) and also Eastern Mediterranean (Egyptian) cultural backgrounds.

For objective two, many of the Egyptian students reported having had different symptoms sometimes/very often during the last 12 months. When comparing our pattern of complaints with students from different European countries that were examined with a similar, but shorter symptom list, some differences were noted (25). First, the most prevalent symptoms in this Egyptian sample were fatigue, mood swings, difficulties to concentrate and headache, while among European students nervousness, headache, back ache and neck/shoulder ache were the leading complaints. Secondly, across most symptoms, the prevalence in our sample was higher (range 15-85\%) than among European students (range 3-62\%) who reported having had the symptom sometimes/very often in the last 12 months (25). It is noteworthy that among European students, students from Turkey and Spain exhibited the highest prevalence suggesting that symptoms may be generally more often reported in countries of the Mediterranean basin as compared to central, Eastern or Northern Europe (25). However, it remains unclear, whether these differences actually reflect real differences in the prevalence of complaints, or if cultural factors contribute to a higher level of 'readiness' to report symptoms/health complaints in these countries. In addition, we found that particularly for the psychological symptoms group and the pains/aches symptoms group, more than two thirds of the sample had experienced $\geq 3$ symptoms, suggesting multiple symptoms are frequent among university students. Such clustering of symptoms has also been reported among university students in Korea and in the UK $(2,39)$.

As regards to objective three, our findings indicated that having a higher frequency of symptoms was associated with poorer subjective health and with poorer quality of life at least for psychological and circulatory/breathing symptoms. We are in agreement with the Korean study, where subjective health was negatively associated with gastrointestinal symptoms among students (2); and with research across seven European countries, where several symptoms were associated with lower quality of life (25).

Previous studies reported higher levels of perceived health complaints among females across adolescents, university students, and the general population $(6,37,40)$. We also found higher level of symptoms among females for psychological and gastrointestinal symptoms, but after controlling for other symptom groups, circulatory/breathing symptoms were less frequent among females.

In this current Egyptian sample, no clear association was noted between smoking, illicit drug/s use or alcohol use and the frequency of health complaints when the statistical significance level was adjusted for multiple testing (Bonferroni adjustment). The reason for the lack of such association might be due to the overall low prevalence of alcohol and other illicit drug/s use (5-10\%) in this sample of students. Given that alcohol is not banned in Egypt, these findings might suggest that it is unlikely that the relatively high level of reported health symptoms could be explained by risky behaviours, or that Egyptian students usually tend to cope with bodily or psychological symptoms by consuming alcohol and other illicit drug/s.
In terms of objective four, whilst across all symptoms groups, the frequency of symptoms increased with the increase in the level of perceived stress, this association remained significant (after controlling for the other symptom groups) only for psychological symptoms. It is plausible that the strongest association between perceived stress and psychological symptoms would be expected, as this symptom group comprised conditions such as depressive mood, anxiety, mood swings, difficulties to concentrate, fear, nightmares, eating disturbances, and insomnia. Others have also reported similar associations $(6,37,41)$. Conversely, researchers also reported a positive association between perceived stress and gastrointestinal complaints (2), a relationship that was not found in our analysis.

In relation to objective five, our sample exhibited differences in levels of psychological symptoms and of perceived stress between faculties. Students enrolled at Physical Education had the lowest risk profile regarding both psychological symptoms and perceived stress, while Veterinary Medicine students showed the highest risk profile. Such differences may be explained not only by differences in the academic/educational aspects (content, course workload/demands, course organisation etc.) between different disciplines of study, but also by the fact that physical activity is generally known to diminish stress effects (42).

This study has limitations. The study is cross-sectional; no causal relationships can be assessed. Self-reported measures estimated the frequency of symptoms (no clinical validations were undertaken). The symptoms list and other questionnaire items were translated from English to Arabic, which may have caused minor problems. Whilst the high Cronbach's alpha of each of the subscales of the symptom groups does not indicate such problems, the low Cronbach's alpha for the perceived stress scale may be due to translation problems or cultural differences in the meanings of the four perceived stress items. Our sample remains a convenience sample from one university in Egypt, and selection bias whereby students with frequent health complaints may be less likely to be present in class when the data was collected, cannot be ruled out. Hence our reported prevalence of symptoms may under-estimate the true morbidity across these young adults. We did not inquire about water-pipe smoking which is prevalent in Egypt, hence the prevalence of smokers may have been underestimated in the current study. Despite the limitations, the study has important strengths as to the best of our knowledge no previous research investigated in detail the relationships between perceived stress and a range of self reported symptoms across large student samples from many faculties in an Arabic speaking eastern Mediterranean Region country of predominantly Muslim faith.

\section{CONCLUSION}

The high prevalence of health complaints such fatigue, mood swings, difficulties to concentrate and headache raise concern and calls for preventive actions at universities. Since different types of complaints have been shown to be associated with psychosocial stress, but also with poor health and poor quality of life, we suggest offering individual counselling and health-oriented courses for students in order to prevent the accumulation of health symptoms and impaired well-being. Several measures such as stress management programmes, a university environment enabling relaxation 
and well-being and the organization of studies and curricula that focus on stress reduction have the potential to decrease perceived stress and psychological symptoms. As students studying physical activity exhibited the lowest risks, an extent of physical activity should be also incorporated in other study programmes.

\section{Acknowledgements}

The authors thank the university, faculties and students who participated in this study. We also acknowledge Drs Kotb S and El-Houfey A.

\section{Conflict of Interest}

None declared

\section{REFERENCES}

1. Brennan G, Shafat A, Mac Donncha C, Vekins C. Lower back pain in physically demanding college academic programs: a questionnaire based study. BMC Musculoskelet Disord. 2007 Jul 13;8:67.

2. Lee EY, Mun MS, Lee SH, Cho HS. Perceived stress and gastrointestinal symptoms in nursing students in Korea: a cross-sectional survey. BMC Nurs. 2011 Nov 8;10:22.

3. Chan GC, Koh D. Understanding the psychosocial and physical work environment in a Singapore medical school. Singapore Med J. 2007 Feb;48(2):166-71

4. Omokhodion FO. Psychosocial problems of pre-clinical students in the University of Ibadan Medical School. Afr J Med Med Sci. 2003 Jun;32(2):135-8.

5. Al-Busaidi Z, Bhargava K, Al-Ismaily A, Al-Lawati H, Al-Kindi R, AlShafaee M, et al. Prevalence of depressive symptoms among university students in Oman. Oman Med J. 2011 Jul;26(4):235-9.

6. Stock C, Kücük N, Miseviciene I, Guillén-Grima F, Petkeviciene J, Aguinaga-Ontoso I, et al. Differences in health complaints among university students from three European countries. Prev Med. 2003 Dec;37(6 Pt 1):535-43.

7. Bigal ME, Bigal JM, Betti M, Bordini CA, Speciali JG. Evaluation of the impact of migraine and episodic tension-type headache on the quality of life and performance of a university student population. Headache. 200 Jul-Aug;41(7):710-9

8. Edberg R, Elasowich B. Student mental health, diminishing resources: two challenges for urban community colleges. Commun Coll Lead 2006;1(7): 1 .

9. American College Health Association. American College Health Association National College Health Assessment (ACHA-NCHA) Spring 2005 Reference Group Data Report (Abridged). J Am Coll Health. 2006 Jul-Aug;55(1):5-16.

10. Gaultney JF. The prevalence of sleep disorders in college students: impact on academic performance. J Am Coll Health. 2010;59(2):91-7.

11. Chrzanowska D, Wdowiak L, Bojar I. The origin of stress, its causes, symptoms and frequency of appearance among the students of Medical University of Lublin. Ann Univ Mariae Curie Sklodowska Med. 2004;59(1):438-43.

12. Fairbrother K, Warn J. Workplace dimensions, stress and job satisfaction. J Manage Psychol 2003;18(1):8-21.

13. Rodrigues RN, Viegas CA, Abreu E Silva AA, Tavares P. Daytime sleepiness and academic performance in medical students. Arq Neuropsiquiatr. 2002 Mar;60(1):6-11.

14. Mesquita G, Reimão R. Quality of sleep among university students: effects of nighttime computer and television use. Arq Neuropsiquiatr. 2010 Oct;68(5):720-5.

15. El Ansari W, Stock C. Is the health and wellbeing of university students associated with their academic performance? Cross sectional findings from the United Kingdom. Int J Environ Res Public Health. 2010 Feb;7(2):509-27.

16. Dusselier L, Dunn B, Wang Y, Shelley MC 2nd, Whalen DF. Personal, health, academic, and environmental predictors of stress for residence hall students. J Am Coll Health. 2005 Jul-Aug;54(1):15-24.

17. El Ansari W, Maxwell AE, Mikolajczyk RT, Stock C, Naydenova V, Krämer A. Promoting public health: benefits and challenges of a Europeanwide research consortium on student health. Cent Eur J Public Health. 2007 Jun;15(2):58-65.
18. El Ansari W, Stock C; UK Student Health Group, Snelgrove S, Hu X, Parke S, Davies J, et al. Feeling healthy? A survey of physical and psychological wellbeing of students from seven universities in the UK. Int J Environ Res Public Health. 2011 May;8(5):1308-23.

19. El Ansari W, Stock C, Mikolajczyk RT. Relationships between food consumption and living arrangements among university students in four European countries - a cross-sectional study. Nutr J. 2012 Apr 24;11:28.

20. El Ansari W, Labeeb S, Kotb S, Yousafzai MT, El-Houfey A, Stock C. Correlates of smoking, quit attempts and attitudes towards total smoking bans at university: findings from eleven faculties in Egypt. Asian Pac J Cancer Prev. 2012;13(6):2547-56.

21. El Ansari W, Stock C. Factors associated with smoking, quit attempts and attitudes towards total smoking bans at university: a survey of seven universities in England, Wales and Northern Ireland. Asian Pac J Cancer Prev. 2012;13(2):705-14.

22. El Ansari W, Sebena R, Stock C. Socio-demographic correlates of six indicators of alcohol consumption: survey findings of students across seven universities in England, Wales and Northern Ireland. Arch Public Health. 2013 Nov 6;71(1):29.

23. El Ansari W, Stock C, Mills C. Is alcohol consumption associated with poor academic achievement in university students? Int J Prev Med. 2013 Oct;4(10):1175-88.

24. Cohen S, Kamarck T, Mermelstein R. A global measure of perceived stress. J Health Soc Behav. 1983 Dec;24(4):385-96.

25. Stock C, Mikolajczyk RT, Bilir N, Petkeviciene J, Naydenova V, Dudziak $\mathrm{U}$, et al. Gender differences in students' health complaints: a survey in seven countries. J Public Health. 2008;16(5):353-60.

26. Hurrelmann K, Kolip P. Survey on the health of school-age children. In: Presseinformationsdienst des SFB 227, No. 11. Bielefeld: University of Bielefeld; 1994. (In German.)

27. Potthoff P, Schroeder E, Reis U, Klamert A. Process and results of field work concerning the Federal Health Survey. Gesundheitswesen. 1999 Dec;61 Spec No:S62-7. (In German.)

28. World Health Organization. Obesity: preventing and managing the global epidemic. Geneva: WHO; 2000.

29. El Ansari W, El Ashker S, Moseley L. Associations between physical activity and health parameters in adolescent pupils in Egypt. Int J Environ Res Public Health. 2010 Apr;7(4):1649-69.

30. Bruusgaard D, Nessiøy I, Rutle O, Furuseth K, Natvig B. Measuring functional status in a population survey. The Dartmouth COOP functional health assessment charts/WONCA used in an epidemiological study. Fam Pract. 1993 Jun;10(2):212-8.

31. Pallant J. SPSS survival manual. Buckingham: Open University Press; 2001

32. Tabachnick BG, Fidell LS. Using multivariate statistics. 3rd ed. New York: Harper Collins; 1996.

33. Nunnally JO. Psychometric theory. New York: McGraw Hill; 1978.

34. Catell RB. The scree test for the number of factors. Multivariate Behav Res. 1966;1(2):245-76.

35. Zwick WR, Velicer WF. Comparison of five rules for determining the number of components to retain. Psychol Bull. 1986 May;99(3):432-42.

36. Tabachnick BG, Fidell LS. Using multivariate statistics. 5th ed. Boston: Allyn and Bacon; 2007.

37. Haugland S, Wold B, Stevenson J, Aaroe LE, Woynarowska B. Subjective health complaints in adolescence. A cross-national comparison of prevalence and dimensionality. Eur J Public Health. 2001 Mar;11(1):4-10.

38. Stock C, Krämer A. The health of students during their education. Gesundheitswesen. 2001 Mar;63 Suppl 1:S56-9. (In German.)

39. El Ansari W, Oskrochi R, Stock C. Symptoms and health complaints and their association with perceived stress: students from seven universities in England, Wales and Northern Ireland. J Public Health. 2013;21(5):413-25.

40. Bingefors K, Isacson D. Epidemiology, co-morbidity, and impact on health-related quality of life of self-reported headache and musculoskeletal pain - a gender perspective. Eur J Pain. 2004 Oct;8(5):435-50

41. Jou YH, Fukada H. Stress and social support in mental and physical health of Chinese students in Japan. Psychol Rep. 1997 Dec;81(3 Pt 2):1303-12.

42. Norris R, Carroll D, Cochrane R. The effects of physical activity and exercise training on psychological stress and well-being in an adolescent population. J Psychosom Res. 1992 Jan;36(1):55-65. 